\title{
A GENERALIZATION OF THE INEQUALITY OF THE ARITHMETIC-GEOMETRIC MEANS
}

by JOHN HUNTER

(Received 14th December, 1954)

\$1. Introduction. The main result in this paper, contained in Theorem 1, is a generalisation of the inequality of the arithmetic-geometric means. A result of a similar character has been proved by Siegel (2). The present result gives an improvement in the inequality in the case when the variables involved are not all distinct, whereas Siegel's result does not. The theorem is used in $\$ 3$ to obtain a result in connection with totally real and positive algebraic integers.

$\S 2$. The main result. Let $x_{1}, \ldots, x_{n}$ be real and positive and write

$$
A=\sum_{i<j}\left(x_{i}-x_{j}\right)^{2}, n s=\sum_{i=1}^{n} x_{i},
$$

and $p=\prod_{i=1}^{n} x_{i}$. Then $A=0$ if and only if $x_{1}=x_{2}=\ldots=x_{n}$, and then $\frac{s^{n}}{p}=1$, since we have assumed each $x_{i}>0$. In what follows we exclude the case $A=0$.

Theorem 1. If $\beta$ denotes the root in the interval $0<\beta<\mathrm{l}$ of the equation

then

$$
\frac{s^{n}}{p} \geqslant \frac{A=\beta^{2}(n-1)(n s)^{2}, \ldots}{\{1+\beta(n-1)\}(1-\beta)^{n-1}} .
$$

We note :

1. Since $A=(n-1)\left(\Sigma x_{i}\right)^{2}-2 n \Sigma x_{i} x_{j}=(n-1)(n s)^{2}-2 n \Sigma x_{i} x_{j}$, we have

$$
0<\frac{A}{(n-1)(n s)^{2}}<1 \text {, }
$$

so that $\beta$ is uniquely determined in the interval $0<\beta<1$.

2. If

$$
f(\beta)=\frac{1}{\{1+\beta(n-1)\}(1-\beta)^{n-1}},
$$

then, in $0<\beta<1$, for $n \geqslant 2, f(\beta)$ is a steadily increasing function of $\beta$. For,

$$
\frac{d f}{d \beta}=\frac{\beta n(n-1)}{\{1+\beta(n-1)\}^{2}(1-\beta)^{n}}>0, \text { for } 0<\beta<1 \text {. }
$$

Also $f(0)=1$, so that $f(\beta)>1$ for $0<\beta<1$.

3. For $n=2$, (1) becomes $\beta^{2}=\frac{\left(x_{1}-x_{2}\right)^{2}}{(2 s)^{2}}=\frac{\left(x_{1}-x_{2}\right)^{2}}{\left(x_{1}+x_{2}\right)^{2}}$, and the right-hand side of (2) equals

$$
\frac{1}{1-\beta^{2}}=\frac{1}{1-\frac{\left(x_{1}-x_{2}\right)^{2}}{\left(x_{1}+x_{2}\right)^{2}}}=\frac{\left(\frac{x_{1}+x_{2}}{2}\right)^{2}}{x_{1} x_{2}}=\frac{s^{2}}{p},
$$

so that the result is true with equality for $n=2$. We can thus assume that $n \geqslant 3$.

For the proof we use the following lemma: 
Lemas l. If

where

$$
\begin{aligned}
& A=\sum_{i<j}\left(x_{i}-x_{j}\right)^{2}, \\
& \sum_{i=1}^{n} x_{i}=n s, \ldots \ldots . . \\
& \prod_{i=1}^{n} x_{i}=p, \ldots \ldots \ldots . . .
\end{aligned}
$$

with

$$
\frac{s^{n}}{p}>1
$$

and

$$
x_{i}>0 \quad(i=1, \ldots, n),
$$

then

$$
A \leqslant n^{2}(n-1) \alpha^{2} s^{2},
$$

where

$$
\{1+\alpha(n-1)\}(1-\alpha)^{n-1}=\frac{p}{s^{n}}, \text { and } 0<\alpha<1 .
$$

For a given set of positive values of $x_{3}, \ldots, x_{n}$, the equations

$$
x_{1}+x_{2}=n s-\left(\sum_{i=3}^{n} x_{i}\right), \quad x_{1} x_{2}=p\left(\prod_{i=3}^{n} x_{i}\right)^{-1}
$$

completely determine $x_{1}, x_{2}$ as the roots of the equation

$$
y^{2}-\left\{n s-\sum_{i=3}^{n} x_{i}\right\} y+p\left(\prod_{i=3}^{n} x_{i}\right)^{-1}=0 .
$$

The values of $x_{1}, x_{2}$ are both positive if.and only if the following two conditions are satisfied

$$
\sum_{i=3}^{n} x_{i}<n s, \quad\left\{n s-\sum_{i=3}^{n} x_{i}\right\}^{2} \geqslant 4 p\left(\prod_{i=3}^{n} x_{i}\right)^{-1} ;
$$

also $x_{1}, x_{2}$ are unequal, except when equality arises in the second condition. The two conditions define a closed domain $D$ in $R_{n-2}$, its points having positive co-ordinates $x_{3}, \ldots, x_{n}$. The boundary of $D$ is given by $x_{1}=x_{2}$.

Now $A$ attains its maximum in $D$, and $A=0$ if and only if $x_{1}=x_{2}=\ldots=x_{n}$, so that at the maximum not all of the $x_{i}$ are equal. Hence, by symmetry, we can suppose that $A$ attains its maximum in $D$ at an inner point of $D$. The values of $x_{i}$ at this maximum must satisfy the equations

for some $\lambda, \mu$;

$$
\frac{\partial A}{\partial x_{i}}+\lambda+\frac{\mu}{x_{i}}=0 \quad(i=1, \ldots, n),
$$

that is

$$
2 n\left(x_{i}-s\right)+\lambda+\frac{\mu}{x_{i}}=0 \quad(i=1, \ldots, n) ;
$$

that is

$$
x_{i}{ }^{2}+\left(\frac{\lambda}{2 n}-s\right) x_{i}+\frac{\mu}{2 n}=0 \quad(i=1, \ldots, n),
$$

and so the $x_{i}$ satisfy a quadratic equation. Hence at the maximum we have $k$ of the $x_{i}$ equal in value to $x$, say, and the remaining $n-k$ of the $x_{i}$ equal to a second number $y$, say, where, by symmetry, $k$ is one of the integers $0,1, \ldots \ldots,\left[\frac{n}{2}\right]$, and where, from (3) and (4),

and

$$
k x+(n-k) y=n s,
$$

$$
x^{k} y^{n-k}=p \text {. }
$$


The corresponding value of $A$ is

$$
A=k(n-k)(x-y)^{2} \text {. }
$$

For $k=0, A=0$ and we require $y=s=p^{\frac{1}{n}}$, i.e. $\frac{s^{n}}{p}=1$, which we have excluded. Thus we have to consider only $k \geqslant 1$. From (6),

Thus (8) becomes

$$
k(x-y)=n(s-y)
$$

$$
\begin{aligned}
A & =k(n-k) \frac{n^{2}}{k^{2}}(s-y)^{2} \\
& =n^{2} s^{2}\left(\frac{n}{k}-1\right)(1-u)^{2}, \quad \text { where } y=u s, \\
& =n^{2} s^{2} \alpha^{2}\left(\frac{n}{k}-1\right), \quad \text { where } \alpha=1-u, \quad .
\end{aligned}
$$

so that $y=(1-\alpha) s$.

Now, from (6) and (5),

$$
0<y<\frac{n}{n-k} s .
$$

Thus

$$
0<1-\alpha<\frac{n}{n-k},
$$

and so

$$
\frac{-k}{n-k}<\alpha<1 \text {. }
$$

From (6),

$$
x=s\left\{\frac{n}{k}-\left(\frac{n}{k}-1\right) u\right\} .
$$

Thus, from (7),

$$
\left\{\frac{n}{k}-\left(\frac{n}{k}-1\right) u\right\}^{k} u^{n-k}=\frac{p}{s^{n}},
$$

and so

$$
\left\{1+\alpha\left(\frac{n}{k}-1\right)\right\}^{k}(1-\alpha)^{n-k}=\frac{p}{s^{n}},
$$

where, by (10), we have to consider the roots of this polynomial in $\alpha$ in the interval

$$
-1 \leqslant \frac{-k}{n-k}<\alpha<1
$$

the -1 arising when $n$ is even, and $k=\left[\frac{n}{2}\right]$.

Let $g(\alpha)=\left\{1+\alpha\left(\frac{n}{k}-1\right)\right\}^{k}(1-\alpha)^{n-k}-\frac{p}{s^{n}}$, and consider this function in the interval $\left[\frac{-k}{n-k}, 1\right]$. We have $g(1)=g\left(\frac{-k}{n-k}\right)=\frac{-p}{s^{n}}$, so that

$$
0>\left\{\begin{array}{c}
g(1) \\
g\left(\frac{-k}{n-k}\right)
\end{array}\right\}>-1 \text {. }
$$

Also $g(0)=1-\frac{p}{s^{n}}$, so that $0<g(0)<1$. Further

$$
g^{\prime}(\alpha)=(n-k)\left\{1+\alpha\left(\frac{n}{k}-1\right)\right\}^{k-1}(1-\alpha)^{n-k-1}\left(-\frac{n}{k} \alpha\right) .
$$

Thus $g^{\prime}(\alpha)=0$ at $\alpha=0, \alpha=1$ (if $n \geqslant 3$ ), and $\alpha=-k /(n-k)$ if $k \geqslant 2$. Also 


$$
g^{\prime}(\alpha)\left\{\begin{array}{lll}
>0 & \text { at } & \alpha=\frac{-k}{n-k} \text { if } k=1, \\
>0 & \text { for } & \frac{-k}{n-k}<\alpha<0, \\
<0 & \text { for } & 0<\alpha<1,
\end{array}\right.
$$

since $k=1,2, \ldots,\left[\begin{array}{l}n \\ \frac{2}{2}\end{array}\right]$. Hence $g(\alpha)$ increases steadily from $g\left(\frac{-k}{n-k}\right)<0$ to $g(0)>0$ as $\alpha$ increases from $\frac{-k}{n-k}$ to 0 , and decreases steadily from $g(0)>0$ to $g(1)<0$ as $\alpha$ increases from 0 to 1 . Thus $g(\alpha)=0$ has one root $-\alpha_{1}$, say, where $\alpha_{1}>0$, in $\frac{-k}{n-k}<\alpha<0$ and one root $\alpha_{2}$, say, in $0<\alpha<1$. We show, by the following lemma, that we need consider only $\alpha_{2}$ in finding the maximum of $A$.

LEMMA 2. If $-\alpha_{1}$ and $\alpha_{2}$ are the roots of the equation

$$
\left\{1+\alpha\left(\frac{n}{k}-1\right)\right\}^{k}(1-\alpha)^{n-k}=\frac{p}{s^{n}}
$$

in the intervals $\frac{-k}{n-k}<\alpha<0$ and $0<\alpha<1$, respectively, where $k=1,2, \ldots,\left[\frac{n}{2}\right]$, then

We have

$$
\alpha_{2} \geqslant \alpha_{1} \text {. }
$$

$$
\left\{1-\alpha_{1}\left(\frac{n}{k}-1\right)\right\}^{k}\left(1+\alpha_{1}\right)^{n-k}=\frac{p}{s^{n}}=\left\{1+\alpha_{2}\left(\frac{n}{k}-1\right)\right\}^{k}\left(1-\alpha_{2}\right)^{n-k}
$$

Since, by above, $g^{\prime}(\alpha)<0$ for $0<\alpha<1$,

$$
\left\{1+\alpha\left(\frac{n}{k}-1\right)\right\}^{k}(1-\alpha)^{n-k}-\frac{p}{s^{n}}>0 \text { for } 0<\alpha<\alpha_{2} .
$$

Thus, to show that $\alpha_{2} \geqslant \alpha_{1}$, it is sufficient to show that

$$
\left\{1+\alpha_{1}\left(\frac{n}{k}-1\right)\right\}^{k}\left(1-\alpha_{1}\right)^{n-k} \geqslant\left\{1+\alpha_{2}\left(\frac{n}{k}-1\right)\right\}^{k}\left(1-\alpha_{2}\right)^{n-k}=\left\{1-\alpha_{1}\left(\frac{n}{k}-1\right)\right\}^{k}\left(1+\alpha_{1}\right)^{n-k}
$$

For simplicity of notation put $m=\frac{n}{k}-1$. Then $k=\frac{n}{m+1}, n-k=\frac{n m}{m+1}$, and $0<\alpha_{1}<\frac{1}{m}$, $1 \leqslant m \leqslant n-1$. We have then to show that

$$
\left\{\left(1+\alpha_{1} m\right)\left(1-\alpha_{1}\right)^{m}\right\}^{\frac{n}{m+1}} \geqslant\left\{\left(1-\alpha_{1} m\right)\left(1+\alpha_{1}\right)^{m}\right\}^{\frac{n}{m+1}},
$$

and so that

$$
\left(1+\alpha_{1} m\right)\left(1-\alpha_{1}\right)^{m} \geqslant\left(1-\alpha_{1} m\right)\left(1+\alpha_{1}\right)^{m},
$$

where $-\alpha_{1}$ is the given root such that $0<\alpha_{1}<\frac{1}{m}$, and $1 \leqslant m \leqslant n-1$.

Let

$$
h(\alpha)=(1+\alpha m)(1-\alpha)^{m}-(1-\alpha m)(1+\alpha)^{m} .
$$

Then

$$
\begin{aligned}
\frac{1}{m(m+1)} h^{\prime}(\alpha) & =\alpha\left\{(1+\alpha)^{m-1}-(1-\alpha)^{m-1}\right\} \\
& >0 \text { for } 0<\alpha \leqslant 1, \text { and so for } 0<\alpha \leqslant \frac{1}{m}, \text { if } m>1, \\
& \equiv 0, \text { if } m=1 .
\end{aligned}
$$

Also $h(0)=0$. Hence $h\left(\alpha_{1}\right)>0$ if $m>1$, and $h\left(\alpha_{1}\right)=0$ if $m=1$. Thus 


$$
\left(1+\alpha_{1} m\right)\left(1-\alpha_{1}\right)^{m} \geqslant\left(1-\alpha_{1} m\right)\left(1+\alpha_{1}\right)^{m},
$$

with equality if and only if $m=1$, and so

giving

$$
\left\{1+\alpha_{1}\left(\frac{n}{k}-1\right)\right\}^{k}\left(1-\alpha_{1}\right)^{n-k}-\frac{p}{s^{n}} \geqslant\left\{1+\alpha_{2}\left(\frac{n}{k}-1\right)\right\}^{k}\left(1-\alpha_{2}\right)^{n-k}-\frac{p}{s^{n}}=0
$$

By this lemma, for each fixed $n$ and $k$,

$$
n^{2} s^{2}\left(\frac{n}{k}-1\right) \alpha_{2}^{2} \geqslant n^{2} s^{2}\left(\frac{n}{k}-1\right) \alpha_{1}^{2} .
$$

Hence, by (9), in finding the maximum of $A$ under the given conditions, we have for each possible $k$, for any given $n$, to consider for $\alpha$ only the unique root in the range $0<\alpha<1$ of the equation

$$
\left\{1+\alpha\left(\frac{n}{k}-1\right)\right\}^{k}(1-\alpha)^{n-k}=\frac{p}{s^{n}} .
$$

We show, by the following lemma, that $k=1$ gives the maximum for each $n$.

Lemma 3. If

$$
u_{k}=\alpha^{2}\left(\frac{n}{k}-1\right)
$$

where $\alpha$ is the root in $0<\alpha<1$ of the equation

$$
\left\{1+\alpha\left(\frac{n}{k}-1\right)\right\}^{k}(1-\alpha)^{n-k}=\frac{p}{s^{n}},
$$

and $k=1,2, \ldots,\left[\frac{n}{2}\right]$, then

$$
\max \left(u_{1}, u_{2}, \ldots, u_{[n / 2]}\right)=u_{1}
$$

As in Lemma 2 we put $m=\frac{n}{k}-1$, so that $k=\frac{n}{m+1}, n-k=\frac{n m}{m+1}$. Then

and so

$$
(1+\alpha m)^{\frac{n}{m+1}}(1-\alpha)^{\frac{m}{m+1} n}=\frac{p}{s^{n}},
$$

$$
(1+\alpha m)^{\frac{1}{m+1}}(1-\alpha)^{\frac{m}{m+1}}=\left(\frac{p}{s^{n}}\right)^{\frac{1}{n}}
$$

where $0<\alpha<1$, and $1 \leqslant m \leqslant n-1$. We have then to consider the maximum of

under these conditions.

$$
U=\alpha^{2} m,
$$

Now

$$
\frac{d U}{d m}=\alpha^{2}+2 \alpha m \frac{d \alpha}{d m}=\alpha\left\{\alpha+2 m \frac{d \alpha}{d m}\right\}
$$

Also, from (11). $\quad \frac{1}{m+1} \log (1+\alpha m)+\frac{m}{m+1} \log (1-\alpha)=\frac{1}{n} \log \frac{p}{s^{n}}$,

Thus

$$
\frac{-1}{(m+1)^{2}} \log (1+\alpha m)+\frac{\alpha}{(m+1)(1+\alpha m)}+\frac{1}{(m+1)^{2}} \log (1-\alpha)+\left\{\frac{1}{1+\alpha m}-\frac{1}{1-\alpha}\right\} \frac{m}{m+1} \frac{d \alpha}{d m}=0 .
$$

Hence

$$
2 m \frac{d \alpha}{d m}=\frac{2(1+\alpha m)(1-\alpha)}{\alpha}\left\{\frac{\alpha}{(m+1)(1+\alpha m)}+\frac{1}{(m+1)^{2}} \log \frac{1-\alpha}{1+\alpha m}\right\} \text {. }
$$


Therefore

Consider

$$
\begin{aligned}
\alpha+2 m \frac{d \alpha}{d m} & =\alpha+\frac{2(1-\alpha)}{m+1}+\frac{2(1+\alpha m)(1-\alpha)}{\alpha(m+1)^{2}} \log \frac{1-\alpha}{1+\alpha m} \\
& =\frac{2(1+\alpha m)(1-\alpha)}{\alpha(m+1)^{2}}\left\{\frac{(1+\alpha m)^{2}-(1-\alpha)^{2}}{2(1+\alpha m)(1-\alpha)}+\log \frac{1-\alpha}{1+\alpha m}\right\}
\end{aligned}
$$

Put

$$
B=\frac{(1+\alpha m)^{2}-(1-\alpha)^{2}}{2(1+\alpha m)(1-\alpha)}+\log \frac{1-\alpha}{1+\alpha m}
$$

$$
t=\frac{1+\alpha m}{1-\alpha} \text {. }
$$

Then $t>1$, since $0<\alpha<1$ and $m \geqslant 1$, and

$$
B=B(t)=\frac{t^{2}-1}{2 t}-\log t=\frac{1}{2}\left(t-\frac{1}{t}\right)-\log t .
$$

Now

$$
\frac{d B}{d t}=\frac{1}{2 t^{2}}(t-1)^{2}>0 \text { for } t>1 \text {, and } B(1)=0 \text {. }
$$

Thus

$$
B>0 \text { for } t>1 \text {. }
$$

Hence, from (13), $\alpha+2 m \frac{d \alpha}{d m}>0$, and so, from (12), $\frac{d U}{d m}>0$, under the given conditions. Hence $\frac{d U}{d k}<0$ and so $U$ is a maximum when $k=1$, which completes the lemma.

By this lemma, $n^{2} s^{2}\left(\frac{n}{k}-1\right) \alpha^{2}$ is a maximum when $k=1, \alpha$ being the unique root in $0<\alpha<1$ of the equation

$$
\left\{1+\alpha\left(\frac{n}{k}-1\right)\right\}^{k}(1-\alpha)^{n-k}=\frac{p}{s^{n}}
$$

that is

$$
\max A=n^{2}(n-1) s^{2} \alpha^{2},
$$

where $\alpha$ is the unique root in $0<\alpha<1$ of the equation

$$
\{1+\alpha(n-1)\}(1-\alpha)^{n-1}=\frac{p}{8^{n}} .
$$

Lemma 1 now follows.

We can now prove Theorem 1. Iet

$$
A_{0}=(n s)^{2}(n-1) \alpha^{2},
$$

where $\alpha$ is the root in $0<\alpha<1$ of the equation

$$
\frac{s^{n}}{p}=\frac{1}{\{1+\alpha(n-1)\}(1-\alpha)^{n-1}}=f(\alpha) .
$$

Let $x_{1}, \ldots, x_{n}$ be an arbitrary set of positive numbers such that $\Sigma x_{i}=n s$, and $\Pi x_{i}=p$. Then, by Lemma 1,

$$
A=\sum_{i<j}\left(x_{i}-x_{j}\right)^{2} \leqslant A_{0}
$$

As noted earlier there is a unique number $\beta$ in $0<\beta<1$ such that

$$
\frac{A}{(n s)^{2}(n-\mathrm{l})}=\beta^{2} \text {. }
$$

But $\frac{A_{0}}{(n s)^{2}(n-1)}=\alpha^{2}$. Hence $\beta \leqslant \alpha$. 
Now $f(\alpha)$ is monotonic increasing in $0<\alpha<1$. Therefore

$$
\frac{s^{n}}{p} \geqslant \frac{1}{\{1+\beta(n-1)\}(1-\beta)^{n-1}},
$$

where $\beta$ is the root in $0<\beta<1$ of the equation

which is the required result.

$$
(n s)^{2} \frac{A}{(n-1)}=\beta^{2}
$$

\$3. Application of Theorem 1. For the application we now suppose that $x_{1}, \ldots, x_{n}$ are the roots of an irreducible polynomial equation $x^{n}+a_{1} x^{n-1}+\ldots+a_{n}=0$, with rational integral coefficients $a_{1}, \ldots, a_{n}$, so that $x_{1}, \ldots, x_{n}$ are the conjugates of a totally real and positive algebraic integer.

Write

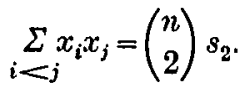

Then

$$
A=(n-1)(n s)^{2}-2 n \sum x_{i} x_{i}=(n-1) n^{2} s^{2}-n^{2}(n-1) s_{2}=n^{2}(n-1)\left(s^{2}-s_{2}\right) .
$$

Theorem 1 can be written :

$$
\frac{s^{n}}{p} \geqslant \frac{1}{\{1+\beta(n-1)\}(1-\beta)^{n-1}},
$$

where $\beta$ is the root in $0<\beta<1$ of the equation

$$
s_{2}=\left(1-\beta^{2}\right) s^{2} .
$$

Since $\stackrel{n}{I} x_{i}=(-1)^{n} a_{n}=p$ is a positive rational integer, $p \geqslant 1$, and so

$$
s^{n} \geqslant \frac{1}{\{1+\beta(n-1)\}(1-\beta)^{n-1}} .
$$

Also

$$
s_{2} \geqslant \frac{1-\beta^{2}}{\{1+\beta(n-1)\}^{\frac{2}{n}}(1-\beta)^{2-\frac{2}{n}}}=\frac{1+\beta}{\{1+\beta(n-1)\}^{\frac{2}{n}}(1-\beta)^{1-\frac{2}{n}}}=f_{1}(\beta),
$$

say. Now, if $n>2$ and $0<\beta<1$,

$$
\frac{d f_{1}}{d \beta}=\frac{2 \beta(n-2)}{\{1+\beta(n-1)\}^{1+\frac{2}{n}}(1-\beta)^{2-\frac{2}{n}}}>0 .
$$

Also

$$
f_{1}(0)=1 \text {. }
$$

Thus $s_{2}>1$ for $0<\beta<1$ if $n>2$; and $f_{1}(\beta)=1$ for $n=2$. Hence $s_{2} \geqslant 1$, which, of course, can be established in other ways.

From a result for $s$ under the present assumptions, obtained by Siegel ((2): Theorem II), wo deduce the corresponding result for $s_{2}$. Siegel's result is :

Let $\theta$ be the positive root of the equation

$$
(1+\theta) \log \left(1+\frac{1}{\theta}\right)-\frac{\log \theta}{1+\theta}=1,
$$

and

$$
\lambda_{0}=e\left(1+\frac{1}{\theta}\right)^{-\theta}
$$


Then, if $\lambda$ is a real number satisfying $1<\lambda<\lambda_{0}=1 \cdot 7336 \ldots$, there is a positive integer $N=N(\lambda)$ such that $s>\lambda$, for all $n \geqslant N$.

We prove :

TheоReм 2. If $\lambda$ satisfies $1<\lambda<\lambda_{0}$, there exists a positive integer $N=N(\lambda)$ such that

We note :

$$
s_{2}>\lambda, \text { for all } n \geqslant N \text {. }
$$

1. In each of the above we can take $\lambda>1$. For, $s \geqslant 1$ and $s_{2} \geqslant 1$, and equality arises (for $n \geqslant 2$ in the case $s=1$ and for $n>2$ in the case $s_{2}=1$ ) if and only if $x_{1}=x_{2}=\ldots=x_{n}$, in which case the equation $x^{n}+a_{1} x^{n-1}+\ldots+a_{n}=0$ is reducible.

2. For every odd prime $p, 4 \cos ^{2} \frac{\pi}{p}$ is a totally real and positivo algebraic integer of degree $n=\frac{1}{2}(p-1)$, the corresponding equation being

$$
x^{n}-(2 n-1) x^{n-1}+(n-1)(2 n-3) x^{n-2}-\frac{1}{3}(n-2)(2 n-3)(2 n-5) x^{n-3}+\ldots=0 .
$$

Thus, for this particular case, $\Sigma x_{i} x_{j}=(n-1)(2 n-3)=\left(\begin{array}{l}n \\ 2\end{array}\right) 4\left(1-\frac{3}{2 n}\right)$, so that $s_{2}=4\left(1-\frac{3}{2 n}\right)$. Hence, if $\mu$ is the best-possible constant in Theorem $2, \lambda_{0} \leqslant \mu \leqslant 4$. It is fairly clear that $\mu>\lambda_{0}$.

Proof of Theorem 2. Choose $\lambda_{1}$ with $\lambda<\lambda_{1}<\lambda_{0}$ and $\epsilon$ in $0<\epsilon<1$ such that $\lambda=\lambda_{1}{ }^{1-\epsilon}$. Now

$$
\begin{aligned}
s_{2}=\left(1-\beta^{2}\right) s^{1+\epsilon} s^{1-\epsilon} & \geqslant \frac{1+\beta}{\{1+\beta(n-1)\}^{\frac{1+\epsilon}{n}}(1-\beta)^{\epsilon-\frac{1+\epsilon}{n}}} s^{1-\epsilon} \\
& =f_{2}(\beta) s^{1-\epsilon}, \ldots \ldots \ldots \ldots \ldots \ldots \ldots \ldots \ldots \ldots \ldots \ldots \ldots \ldots \ldots \ldots \ldots
\end{aligned}
$$

where

$$
f_{2}(\beta)=\frac{1+\beta}{\{1+\beta(n-1)\}^{\frac{1+\epsilon}{n}}(1-\beta)^{\epsilon-\frac{1+\epsilon}{n}}} .
$$

Take $N_{1}$ such that $\epsilon-\frac{1+\epsilon}{n}>0$, that is $\epsilon>\frac{1}{n-1}$, for $n \geqslant N_{1}$. For such an $n$ consider $f_{2}(\beta)$ in $0<\beta<1$.

$$
\frac{d f_{2}}{d \beta}=\frac{\beta(n-1)(1-\epsilon)}{\{1+\beta(n-1)\}^{1+\frac{1+\epsilon}{n}}(1-\beta)^{1+\epsilon-\frac{1+\epsilon}{n}}}\left\{(1-\beta)+\frac{2}{1-\epsilon}\left(\epsilon-\frac{1}{n-1}\right)\right\}>0
$$

in $0<\beta<1$, since $\epsilon-\frac{1}{n-1}>0$. Now $f_{2}(0)=1$. Thus $f_{2}(\beta)>1$ in $0<\beta<1$. Hence, from (15),

By Siegel's result,

$$
s_{2}>s^{1-\epsilon}, \quad \text { for } n \geqslant N_{1} \text {. }
$$

Thus

$$
s>\lambda_{1}, \quad \text { for } n \geqslant N_{2} \text {, say. }
$$

that is

$$
\begin{array}{ll}
s_{2}>\lambda_{1}^{1-\epsilon} & \text { for } n \geqslant N=\max \left(N_{1}, N_{2}\right) ; \\
s_{2}>\lambda & \text { for } n \geqslant N .
\end{array}
$$

§4. Inequalities for $\frac{A}{n^{2}}$ and $\frac{\Sigma x_{i}^{2}}{n}$. An inequality for $\frac{A}{n^{2}}=\frac{1}{n^{2}} \underset{i<j}{\sum}\left(x_{i}-x_{j}\right)^{2}$ can be deduced from a result due to Schur (1). The inequality can be stated in the form :

If $x_{1}, \ldots, x_{n}$ are the conjugates of a totally real algebraic integer, and if $\lambda$ is such that

$$
0<\lambda<e^{\dagger}=1.6487 \ldots,
$$

then there is an integer $N=N(\lambda)$ such that $\frac{A}{n^{2}}>\lambda$ for $n \geqslant N$.

For completeness we include a proof of this result. The proof is based on the following lemmas : 
LEMMA 4. If $\Delta\left(x_{1}, \ldots, x_{n}\right) \equiv \prod_{i<j}\left(x_{i}-x_{j}\right)^{2}$, where the $x_{i}$ are real numbers such that $\sum_{i=1}^{n} x_{i}^{2} \leqslant B$, then This follows from $\operatorname{Schur}(1, \S 2)$.

$$
\max \Delta\left(x_{1}, \ldots, x_{n}\right)=R_{n}\left(\frac{B}{n^{2}-n}\right)^{\frac{1}{\left(n^{2}-n\right)}} \text {, where } R_{n}=\prod_{m=1}^{n} m^{m} \text {. }
$$

\section{LEMMA 5.}

$$
R_{n}=\left(\frac{n}{e t}\right)^{\ddagger\left(n^{2}-n\right)} n^{n+r^{\prime}:} \exp \left\{-\frac{1}{4} n+O(1)\right\} .
$$

This can be obtained by applying the Euler summation formula

$$
\sum_{m=1}^{n} f(m)=\frac{1}{2}\{f(n)-f(0)\}+\int_{0}^{n} f(x) d x+\int_{0}^{n} f^{\prime}(x)\left(x-[x]-\frac{1}{2}\right) d x
$$

to the function $f(x)=x \log x$.

Now $\frac{A}{n^{2}}=\frac{1}{n^{2}} \sum_{i<j}\left(x_{i}-x_{j}\right)^{2}=\frac{1}{n^{2}}\left\{n \sum x_{i}{ }^{2}-\left(\Sigma x_{i}\right)^{2}\right\}=\frac{1}{n} \sum_{i=1}^{n}\left(x_{i}-s\right)^{2} . \quad$ By Lemma $4, \sum_{i=1}^{n}\left(x_{i}-s\right)^{2} \leqslant n \lambda$ implies $\Delta\left(x_{1}-s, \ldots, x_{n}-s\right) \leqslant R_{n}\left(\frac{n \lambda}{n^{2}-n}\right)^{1\left(n^{2}-n\right)}$. Now $\Delta\left(x_{1}-s, \ldots, x_{n}-s\right)=\Delta\left(x_{1}, \ldots, x_{n}\right) \geqslant\left(\frac{n^{n}}{n !}\right)^{2}$, by Minkowski's discriminant inequality. Hence

$$
1 \leqslant\left(\frac{n !}{n^{n}}\right)^{2} R_{n}\left(\frac{n \lambda}{n^{2}-n}\right)^{\frac{1}{\left(n^{3}-n\right)}}
$$

Thus, by Lemma 5 and Stirling's approximation for $n !$,

$$
1<C e^{-\frac{7 n}{4} n^{n+\frac{13}{12}}}\left(\frac{\lambda}{e^{\frac{1}{t}}}\right)^{1\left(n^{2}-n\right)},
$$

where $C$ is a constant. Since $\frac{\lambda}{e^{-1}}<1$, the right-hand side of $(16)$ is less than 1 for sufficiently large $n$. Hence (16) implies that $n<N=N(\lambda)$, say. Thus

and so

$$
\frac{1}{n} \sum_{i=1}^{n}\left(x_{i}-s\right)^{2}>\lambda \text { for } n \geqslant N
$$

In the earlier notation, this result can be expressed in the form

and in the form

$$
\begin{aligned}
& (n-1)\left(s^{2}-s_{2}\right)>\lambda \text { for } n \geqslant N \text {, } \\
& (n-1) \beta^{2} s^{2}>\lambda \text { for } n \geqslant N \text {. }
\end{aligned}
$$

We note that the result holds, in particular, when $x_{1}, \ldots, x_{n}$ are the conjugates of a totally real and positive algebraic integer. By (14), the best-possible constant in this case is $\mu$, where $1 \cdot 6487 \ldots \leqslant \mu \leqslant 2$.

In the totally real and positive case we deduce :

If $x_{1}, \ldots, x_{n}$ are the conjugates of a totally real and positive algebraic integer and if $\lambda$ is such that $1<\lambda<e^{1}+\lambda_{0}{ }^{2}=4.654 \ldots$, then there is an integer $N=N(\lambda)$ such that $\frac{\Sigma x_{i}{ }^{2}}{n}>\lambda$ for $n \geqslant N$.

Write $\lambda=\lambda_{1}+\lambda_{2}^{2}$, where $0<\lambda_{1}<e^{t}$ and $1<\lambda_{2}<\lambda_{0}$. We have

$$
\frac{\Sigma x_{i}^{2}}{n}=\frac{A}{n^{2}}+\left(\frac{\Sigma x_{i}}{n}\right)^{2} \text {. }
$$


Now $\frac{A}{n^{2}}>\lambda_{1}$ for $n \geqslant N_{1}$, say,

and, by Siegel's result, $\frac{\sum x_{i}}{n}>\lambda_{2}$ for $n \geqslant N_{2}$, say.

Hence

$$
\frac{\sum x_{i}^{2}}{n}>\lambda \quad \text { for } n \geqslant \max \left(N_{1}, N_{2}\right) \text {. }
$$

Schur gave this result with $e^{t}+e=4 \cdot 367 \ldots$ in place of $4.654 \ldots$ By (14), the best-possible constant in this case is $\mu$, where $4 \cdot 654 \ldots \leqslant \mu \leqslant 6$.

\section{REFERENCES}

(1) I. Schur, "Über die Vorteilung der Wurzoln bei gewissen algebraischen Gleichungen mit ganzzahligen Koeffizienten," Math. Zeitschrifı, 1 (1918), 377-402.

(2) C. L. Siegel, "The trace of totally positive and real algebraic integers," Annals of Math., (2) 46, (1945), 302-312.

The University

GLasgow 